\title{
Automated detection of surface changes on comet 67P
}

\author{
Jean-Baptiste Vincent ${ }^{1}$, Sandor Kruk ${ }^{2}$, Lida Fanara ${ }^{1}$, Samuel Birch ${ }^{3}$, and Abhinav Jindal ${ }^{4}$ \\ ${ }^{1}$ DLR Institute for Planetary Research, Berlin, Germany (jean-baptiste.vincent@dlr.de) \\ ${ }^{2}$ ESA Scientific Support Office, ESTEC, Noordwijk, The Netherlands \\ ${ }^{3}$ Department of Earth, Atmospheric and Planetary Sciences, Massachusetts Institute of Technology, Cambridge, USA \\ ${ }^{4}$ Cornell University, Department of Astronomy, USA
}

The ejection of cometary material is driven by the sublimation of volatiles, and depends on heliocentric distance, local illumination conditions, and material properties. A short period comet like $67 \mathrm{P}$ loses $0.1 \%$ of its mass per orbit [1], which would amount to $50 \mathrm{~cm}$ thick layer of material, if the loss was uniformly spread across the surface. ESA's Rosetta mission followed comet 67P for more than 2 years, monitoring the surface and its evolution through different seasons and heliocentric distances (4-1.2 AU). One of Rosetta's major discoveries was that a significant amount of material is being displaced rather than expelled [2]. Dust is transported from one region to another, or reorganized in place, leading to a local dust cover which appears to expand and contract with seasons. Sporadic events, sometimes linked to outbursts, create more drastic transformations of the surface: e.g. cliff collapses, sudden formation of deep pits, or rapid expansion of surface depressions.

The large scale changes have been described by many authors [3-9], as the link between morphology and activity $[10,11]$. However, an exhaustive catalog of changes at all scales remain to be published. This is a challenging task, because most changes are small (meter-size or less), which means the associated surface features occupy only a few pixels in the high-resolution images (OSIRIS NAC [12]).

Yet, identifying all surface changes remains necessary if we want to understand how cometary activity and surface evolution are interlinked. An exhaustive catalog of changes would allow for precise calculations of the thermal energy distribution and presence of volatile material in the subsurface, for instance by tracking the displacement of a many small boulders in a given area.

In this work, we present a simple, but efficient approach to accurately identify areas that have changed over time. This an improved version of the study presented in [13]:

- Images of the same area acquired at different epochs are projected onto the tri-dimensional shape model of the comet. We use the software shapeViewer [14]: a mission/instrument simulator. An inverse ray-tracing algorithm maps each facet of the shape model in the current field of view to the CCD pixels, and uses this information to texture the shape with the relevant data. Each image thus projected can be viewed from any other angle, including the viewing geometry of the other image in the pair currently investigated. This effectively co-registers the data, within the uncertainties of the shape model and attitude/orbit information.

- We calculate the density of information in matching sub-areas of the before/after image pair, 
with information being the measure of entropy in pixel values, as introduced in [15]. We also propose an alternative method which relates information to the 2D-Laplacian operator at every pixel. In either case, information does not depend on the absolute value of each pixel, but on how the signal varies from one pixel to the next. This makes our method robust against changes in global illumination between images.

- After the second step, we know how the spatial distribution of information density has changed between the two epochs, at different resolutions. This allow us to quantify not only the extent of local area that has been modified, but also whether this was a positive or negative change, An increase in information density typically indicates the presence of new morphological features. Conversely, a loss of information indicates that surface features have been removed, often the signature of dust deposition.

Our change detection method can be completed by other image processing techniques. For instance if we identify areas prone to boulder movement, one can use advanced feature recognition to track the distribution of those objects automatically. We applied an automated block fall detection method that was developed for estimating the erosion rate of the north polar steep scarps on Mars [16, 17]. This method combines blob detection and machine learning to detect and delineate newly appeared small boulders against complex backgrounds with a high confidence level. A Support Vector Machine, which is trained on Histograms of Oriented Gradients (HOG) of thousands of boulder and non-boulder image samples, identifies the location of new boulders. The blob detection part of the method verifies the existence of the boulders by looking for convex objects that are accompanied by a shadow and delineates their boundaries.

We are now in the process of finalizing the detection of changes on 67P, using at least one image pair per region. While this a major step forward, it is not sufficient. To cover the entire comet and produce an exhaustive list of morphological changes we will be launching a citizen science project, Rosetta Zoo, on the Zooniverse platform. Having volunteers peruse OSIRIS images will allow us to differentiate physical changes in the surface of the comet from spurious detections over the entire dataset. This citizen science project will involve thousands of members of the public in the analysis of images from this cornerstone ESA mission.
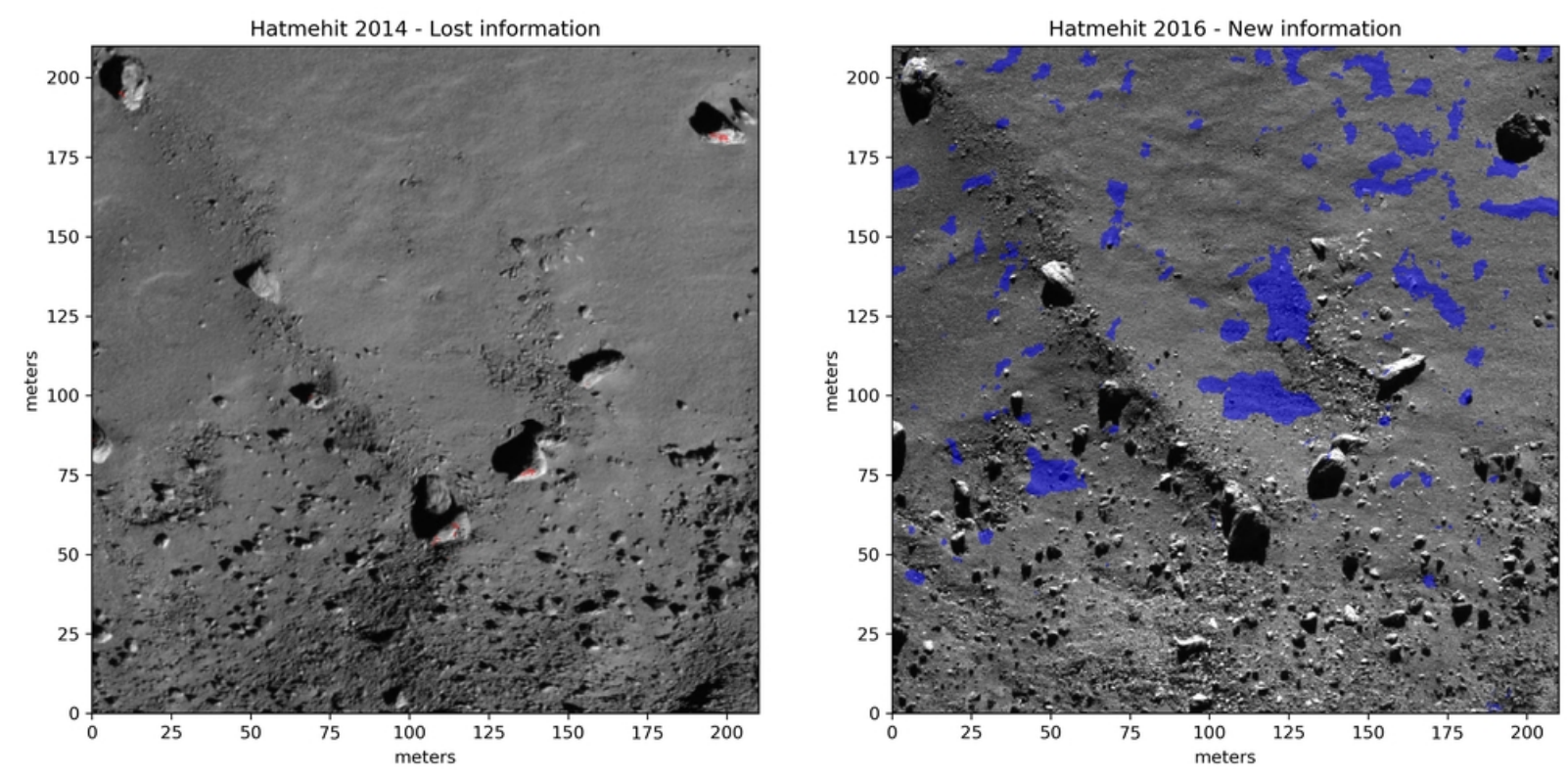

Changes detected in 67P's Hatmehit region between 2014 (left) and 2016 (right). Our algorithm detects that $6.5 \%$ of the area in the second image contains new morphological features (in blue), mostly boulders being exposed after the dust cover has been removed by cometary activity. Data: OSIRIS NAC, images N20140914T165658730ID20F41 \& N20160430T090037998ID20F41 
References: [1] Pätzold et al, Nature (2016); [2] Thomas et al, A\&A (2015); [3] Groussin et al, A\&A (2015); [4] El Maarry et al, Science (2017); [5] Vincent et al, MNRAS (2017); [6] Birch et al, MNRAS (2017); [7] Pajola et al, NatAstro (2017); [8] Birch et al, GRL (2019); [9] Vincent et al, EPSC (2019); [10] El Maarry et al, ISSI/SSR (2020); [11] Vincent et al, ISSI/SSR (2020); [12] Keller et al, SSR (2007); [13] Vincent et al, EPSC (2019); [14] Vincent et al, LPSC (2018); [15] Shannon et al, Bell System Technical Journal (1948); [16] Fanara et al., PSS (2020); [17] Fanara et al., Icarus (2020) 